\title{
Relaxations in relation to Liquid Crystalline Formation and Glass Transition for Comb-like Polymer: Structural Relaxation as expressed by Entropy Model and Activation Energy Spectrum
}

\author{
Yutaka Tanaka* \\ Department of Material Science and Engineering, Faculty of Engineering \\ University of Fukui, Fukui 910-8507, Japan. Email: tanaka@matse.u-fukui.ac.jp
}

\begin{abstract}
This study demonstrates that the modelling approach for the kinetic phenomenon of glass transition is effective to examine the relaxation. In the enthalpy relaxation of poly(cyanobiphenyl-yloxy) alkyl acrylate, the decrease in enthalpy was measured as a function of ageing time and ageing temperature. Obtained data was analysed at first with the activation energy spectrum (AES) model, and next, with the framework of the entropy model which follows the evolution of the configurational entropy of the sample during the whole thermal history in the experiment. The prediction of DSC curve based on the result of analysis for the glass transition will be presented. AES model gives information about thermally activated processes which are available to contribute to observed enthalpy changes against the thermal history. The activated processes are relevant to the rearrangement of molecules. The rearrangement corresponds to the structural changes of polymer chain which can be observed in our study as the change in optical texture, known as the coarsening dynamics of nematic domain for the liquid crystalline materials. The analysis for the coarsening is also shown to elucidate the specific feature of physical parameter.
\end{abstract}

\section{Introduction}

The physical properties of the comb-like polymer are currently attracting much interest in many research works. In particular, the comb-like polymer shows significant variations in the mechanical, optical and thermal properties in the out-of-equilibrium system, where the structural relaxation is the key role to those physical variations. Furthermore, because the structural relaxation usually accompanies dynamics in molecular scale, it is attracting in many research works from the viewpoint of the relationship between the structure and properties. The experimental results shown in this study are concerned with significant variations in optical texture and heat capacities as the relaxation phenomena. ${ }^{(1)}$ Detailed models with the configurational entropy for those variations are applied to interpret in both molecular level and macroscopic level.

Polymer glasses, in themselves, are essentially thermodynamic non-equilibrium states. Structure and properties change with thermal ageing in the state below the glass transition temperature after a rapid change in thermodynamic conditions. Those changes have been investigated for a comb-like polymer with cyanobiphenyl groups in the side chain; it turns out that the enthalpy relaxation is observed clearly for poly cyanobiphenyl acrylate (PCBnA, $\mathrm{n}$ is the alkyl chain length between the backbone and cyanobiphenyl group). To make a better understanding, the activation energy spectrum model (AES model) was applied for quantitative characterisation of the relationship between the relaxation and its molecular mechanism.

\section{* Corresponding author}



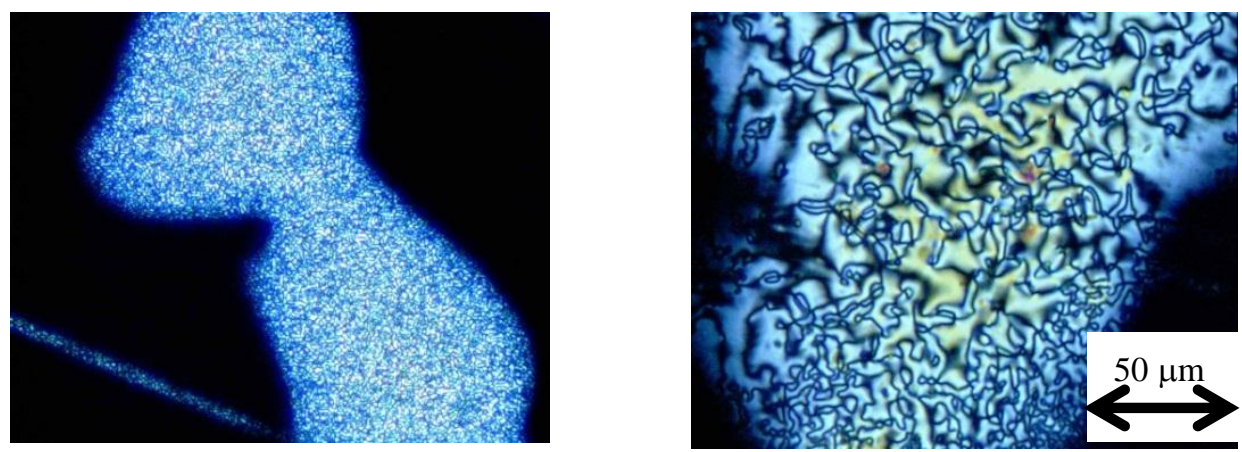

Fig. 1: Polarising optical microscopy images of the magnification of $\times 500$ taken at $25^{\circ} \mathrm{C}$ for $\mathrm{PCB} 2 \mathrm{~A}$ before(left) and after(right) the thermal ageing; A typical example of the structural relaxation upon ageing.

AES model was originally developed for relaxation in metallic or oxide glasses. However, it is applicable to any amorphous material and gives information about thermally activated processes which are available to contribute to observed changes in the properties upon relaxation. In the transition to the equilibrium, any thermally activated rearrangement of single atoms or atom groups plays a key role. The rearrangement corresponds to the structural changes of the polymer chains, which often cause the optical texture to vary in liquid crystalline polymer. The variation is also observed for PCB2A(i.e. $n=2)$, which shows nematic phase, in response to the variation of thermal history as shown in Fig.1. The photographs in Fig. 1 were taken by a polarising optical microscope (POM) for the single sample before and after the thermal ageing. They clearly show the change of the size of the nematic domain, which is an example of relaxation phenomena.

\section{Experimental}

The monomer was synthesized following literature procedures. The polymers of $n=2$ and $n=6$ were prepared by radical polymerisation, then fractionated by GPC. In the DSC experiment of PCB6A, the following thermal program was imposed; starting from the temperature $(T)$ of $160^{\circ} \mathrm{C}$, well above the clearing temperature, the sample was cooled to a specified ageing temperature $\left(T_{\mathrm{A}}\right)$, then the ageing was carried out. After a given ageing time $\left(t_{\mathrm{A}}\right)$, DSC heating curve was obtained at a rate of $5{ }^{\circ} \mathrm{C} / \mathrm{min}$, for the temperature range from -20 to $160^{\circ} \mathrm{C}$ to determine the enthalpy loss of $\Delta H\left(t_{\mathrm{A}}, T_{\mathrm{A}}\right)$, which has the relation with the relaxation function of $\phi$ as shown below.

$$
\Delta H\left(t_{\mathrm{A}}, T_{\mathrm{A}}\right)=\Delta H\left(\infty, T_{\mathrm{A}}\right) \times(1-\phi)
$$

$\Delta H\left(\infty, T_{\mathrm{A}}\right)$ is the value of the limit of $t_{\mathrm{A}} \rightarrow \infty$ for $\Delta H\left(t_{\mathrm{A}}, T_{\mathrm{A}}\right)$. The following expression was taken into account as the relaxation function.

$$
\phi=\exp \left[-\left(t_{\mathrm{A}} / \tau\right)\right]^{\beta}
$$

The relaxation time, $\tau$, and the shape parameter $\beta$ characterise this function.

\section{Results and Discussion}

The glass transition temperature and the nematic-isotropic transition temperature were determined as the usual manner. The variations of the transition temperatures with the molecular weight of PCBnA are approximately consistent with earlier works carried out for the cyanobiphenyl polymer with different 
alkyl chain length; the results were interpretable without difficulty. Contrastively, the isotropic-nematic transitions of PCB2A were not simple. In the cooling DSC scans with a rate of $1{ }^{\circ} \mathrm{C}$, the exothermic peaks at the isotropic-nematic transition were different for different molecular weights. For PCB2A sample of lower molecular weight, the exothermic peak appeared at the transition temperature $\left(T_{\mathrm{N}-\mathrm{I}}\right)$ of $100^{\circ} \mathrm{C}$, while the sample of higher molecular weight showed no peak around $T_{\mathrm{N}-\mathrm{I}}$. In addition, the shoulder of DSC curve was observed concerning the glass transition at around $75^{\circ} \mathrm{C}$ for the sample of lower molecular weight. However, DSC curve of the sample of higher molecular weight did not show endothermic variation around the glass transition temperature $\left(T_{\mathrm{g}}\right)$, and even below $T_{\mathrm{g}}$.

The formation of nematic phase for the sample of low molecular weight was followed by POM observation with the temperature jump from $110^{\circ} \mathrm{C}$ to $95^{\circ} \mathrm{C}$. As a result, it took more than $1 \mathrm{~h}$ that the nematic phase of the sample appeared with the refractive ray under the POM observation. Such a slow dynamics induced by the increase in molecular weight can be relevant to the coarsening dynamics observable for the nematic phase of PCBnA.

Fig. 2 shows the results of DSC experiments for PCB6A sample of $T_{\mathrm{A}}=33^{\circ} \mathrm{C}$ with different ageing time. The raw DSC data was converted to the heat capacity curve of $c_{\mathrm{p}}(T)$ with the calibration using the reference scan of Indium. As the increase in $t_{\mathrm{A}}$, DSC overshoot becomes more pronounced, which shows the enthalpy loss during the isothermal ageing. $\Delta H\left(t_{\mathrm{A}}, T_{\mathrm{A}}\right)$ can be estimated from the area bounded by the DSC curves with and without ageing. As a result, the temperature of the DSC overshoot shifted higher with $t_{\mathrm{A}}$. Because the temperature of the peak corresponds to the process of recovery of enthalpy during the heating of the aged sample, the increase of the overshoot temperature is due to the decrease of the molecular mobility of polymer chain.

According to eq.(1), values of $\phi$ were evaluated and plotted against $t_{\mathrm{A}}$, then $\tau$ and $\beta$ were determined on the basis of eq.(2). And further, the activation energy spectrum was constructed from the relaxation function. Specific manipulations to derive AES have already been described in the earlier works. ${ }^{(2)}$ Fig. 3 shows AES of PCB6A of fractionated sample. It expresses the relation between $P(E)$ and the activation energy of $E ; P(E)$ is the enthalpy change related to relaxation processes having activation energies in the range $(E+\mathrm{d} E)$, it corresponds to the number density of processes. Therefore, the spectrum gives information about the processes controlling the relaxation. In addition, the peak top of AES was revealed to correlate with the energy barrier constant which appears in the configurational entropy model.

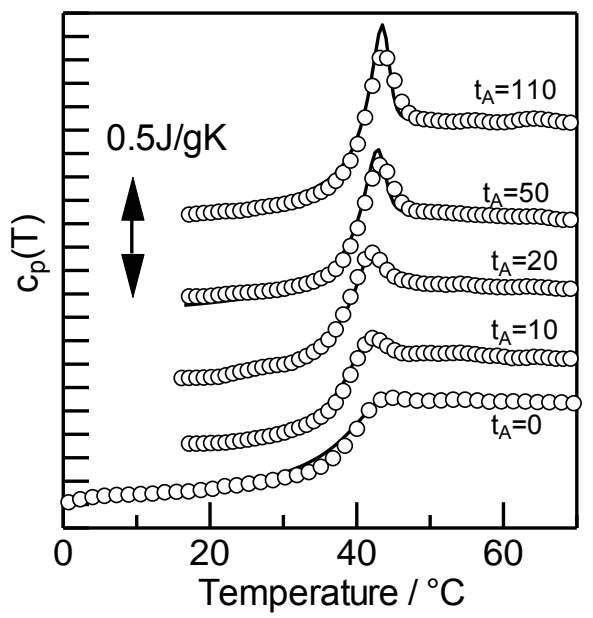

Fig. 2: $\quad c_{p}(T)$ curves (indicated by open circles, $T_{A}=306 K\left(33^{\circ} \mathrm{C}\right)$ ) obtained by DSC measurement for different ageing time $\left(t_{A} / \mathrm{min}\right)$. The solid lines represent the calculation curves obtained from the framwork of $c_{p}(T)$ prediction. 

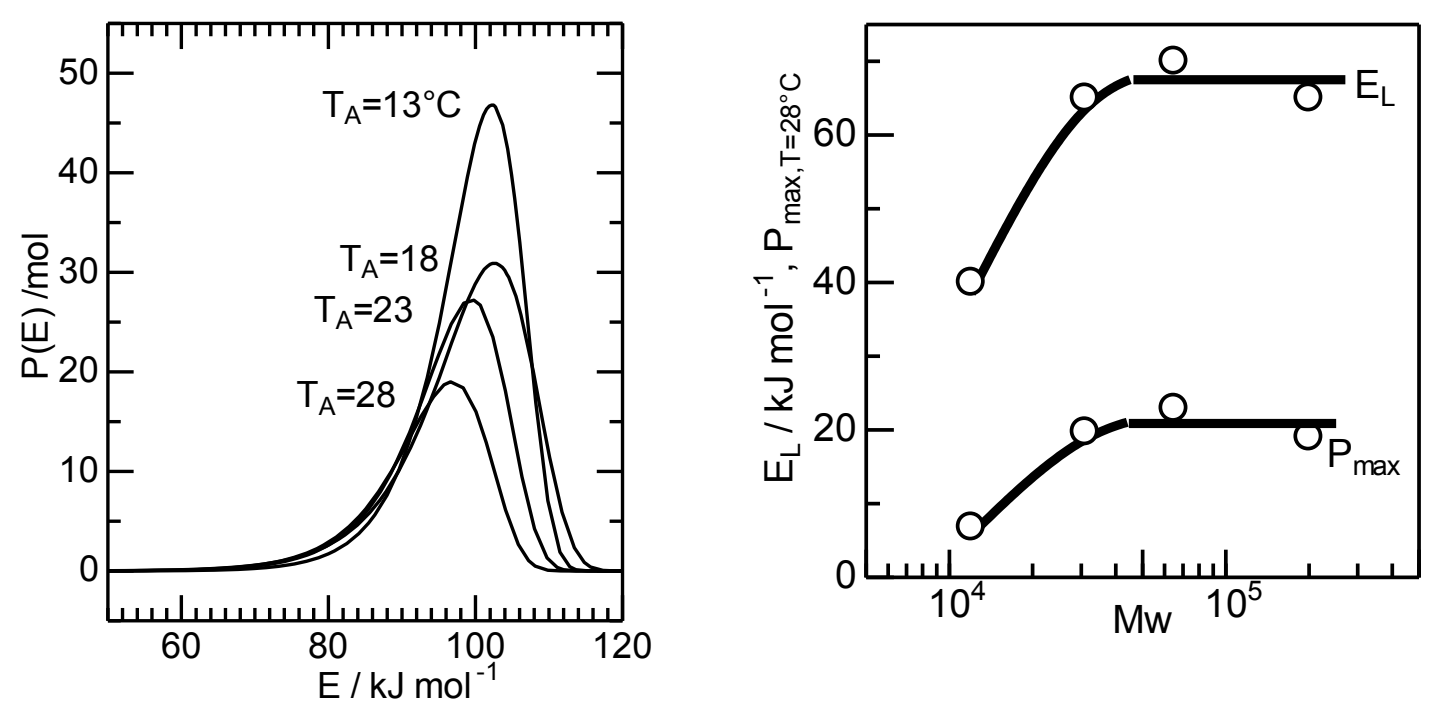

Fig. 3 (left): AES for PCB6A of a fractionated sample $\left(M_{w}=3.1 \times 10^{4}\right) \quad$ The ageing temperatures $\left(T_{A}\right)$ were indicated in the figure. Fig. 4 (right): Cooling DSC curves (rate $\left.=1^{\circ} \mathrm{C} / \mathrm{min}\right)$ for PCB2A samples. The molecular weight of the samples are; PCB2A_l $=1.5 \times 10^{5}, \quad$ PCB2A_2 $=2.7 \times 10^{4}, P C B 2 A \_3=$ $1.7 \times 10^{4}$.

It is clear that almost all the relaxation processes locate within the energy range of the AES. In other words, there are both the lower and the upper limits in the activation energies of the processes. Fig. 4 shows the relationships between the characteristic values of AES of PCB6A and the molecular weight $\left(M_{\mathrm{w}}\right) . \quad E_{\mathrm{L}}$ is the lower limit in the energy range of AES, $P_{\text {max }}$ is the value of the peak maximum for AES of $T_{\mathrm{A}}=28^{\circ} \mathrm{C}$. The plateaus are seen at higher $M_{\mathrm{w}}$ for these values. It is normal behaviour that the plateau value appears at higher $M_{\mathrm{w}}$ for the glass transition.

Next, the framework to predict $c_{\mathrm{p}}(T)$ curve was constructed according to the method of Moynihan, et al with the configurational entropy model. ${ }^{(3)}$ The basic feature of the framework of $c_{\mathrm{p}}(T)$ prediction is the combination between the relaxation functions of infinitesimal intervals and Boltzmann superposition principle. The modelling approach for the enthalpy relaxation of PCBnA enables us to reproduce the DSC curve by calculation alone (independently from the experiment) using the configurational entropy of the sample. The results of the calculation curve for PCB6A were shown in Fig. 2 along with the experimental data. The prediction power is sufficient to reproduce them as shown in the figure. Consequently, the combination between AES and the method of Moynihan with the configurational entropy gives effective analyses for the structural relaxation of the comb-like polymer.

\section{References}

1. Tanaka, Y. Polym. J. 2009, 41, 125-131.

2. Tanaka, Y. Polym. J. 2007, 39, 1030-1039.

3. Moynihan, C. T.; Easteal, A. J.; DeBolt, M. A.; J. Tucker, J. Am. Ceramics Soc. 1976, 59, 12-16; DeBolt, M. A.; Easteal, A. J.; Macedo, P. B.; Moynihan, C. T. ibid 1976, 59, 16-21. 